\title{
AN IMPLICIT WEIGHTED DEGREE CONDITION FOR HEAVY CYCLES ${ }^{1}$
}

\author{
JUNQING CAI \\ School of Management, Qufu Normal University \\ Rizhao, 276826, China \\ e-mail: caijq09@163.com \\ $\mathrm{HAO} \mathrm{LI}^{2}$ \\ Institute for Interdisciplinary Research \\ Jianghan University, Wuhan, 430019, China \\ LRI, UMR 8623, CNRS and Université de Paris-Sud 11 \\ F-91405 Orsay, France \\ e-mail: li@lri.fr \\ AND

\section{WANTAO Ning} \\ Department of Mathematics and Statistic, Xidian University \\ Xi'an, Shaanxi 710071, China \\ e-mail: ningwt05@163.com
}

\begin{abstract}
For a vertex $v$ in a weighted graph $G, i d^{w}(v)$ denotes the implicit weighted degree of $v$. In this paper, we obtain the following result: Let $G$ be a 2-connected weighted graph which satisfies the following conditions: (a) The implicit weighted degree sum of any three independent vertices is at least $t$; (b) $w(x z)=w(y z)$ for every vertex $z \in N(x) \cap N(y)$ with $x y \notin E(G)$; (c) In every triangle $T$ of $G$, either all edges of $T$ have different weights or all edges of $T$ have the same weight. Then $G$ contains either a hamiltonian cycle or a cycle of weight at least $2 t / 3$. This generalizes the result of Zhang et al. [9].
\end{abstract}

Keywords: weighted graph, hamiltonian cycles, heavy cycles, implicit degree, implicit weighted degree.

2010 Mathematics Subject Classification: 05C38.

\footnotetext{
${ }^{1}$ This paper is partially supposed by the Scientific Research Foundation for Doctors in Qufu Normal University (2012015) and NNSF of China (11326218).

${ }^{2}$ Corresponding author.
} 


\section{REFERENCES}

[1] J.A. Bondy, Large cycles in graphs, Discrete Math. 1 (1971) 121-132. doi:10.1016/0012-365X(71)90019-7

[2] J.A. Bondy and U.S.R. Murty, Graph Theory with Applications (MacmillanLondon, Elsevier-New York, 1976).

[3] V. Chvátal and P. Erdős, A note on hamiltonian circuits, Discrete Math. 2 (1972) 111-113. doi:10.1016/0012-365X(72)90079-9

[4] G.A. Dirac, Some theorems on abstract graphs, Proc. Lond. Math. Soc. 2 (1952) 69-81.

[5] H. Enomoto, J. Fujisawa and K. Ota, A $\sigma_{k}$ type condition for heavy cycles in weighted graphs, Ars Combin. 76 (2005) 225-232.

[6] I. Fournier and P. Fraisse, On a conjecture of Bondy, J. Combin. Theory (B) 39 (1985) $17-26$. doi:10.16/0095-8956(85)90035-8

[7] P. Li, Implicit weighted degree condition for heavy paths in weighted graphs, J. Shandong Univ. (Nat. Sci.) 18 (2003) 11-13.

[8] L. Pósa, On the circuits of finite graphs, Magyar Tud. Akad. Mat. Kutató Int. Közl 8 (1963) 355-361.

[9] S. Zhang, X. Li and H. Broersma, A $\sigma_{3}$ type condition for heavy cycles in weighted graphs, Discuss. Math. Graph Theory 21 (2001) 159-166. doi:10.7151/dmgt.1140

[10] Y. Zhu, H. Li and X. Deng, Implicit-degrees and circumferences, Graphs Combin. 5 (1989) 283-290. doi:10.1007/BF01788680

Received 31 October 2011

Revised 18 November 2013

Accepted 18 November 2013 